\title{
Ion Formation from Charged Droplets: Roles of Geometry, Energy, and Time
}

\author{
John B. Fenn \\ Department of Chemical Engineering, Yale University, New Haven, Connecticut, USA
}

\begin{abstract}
The formation of ions from the charged droplets produced in the several spray ionization techniques is viewed as an activated rate process involving field-assisted desorption, in accord with the ideas first set forth by Iribarne and Thomson. The novel features of the present treatment are particularly relevant to the unique ability of electrospray ionization to transform large molecules in solution to free ions in the gas phase, with extensive multiple charging. These new features stem mainly from the realization that the spacing of charges on a desorbed ion must relate to the spacing of charges on the surface of the droplet whence it came. The consequences of this "rule" can account for the existence of maxima and minima in the number of charges on the ions of a particular species as well as the nature of the distribution of ions among the intervening charge states. They also explain the dependence of charge state on the configuration in solution of the parent molectule of the desorbed ion. In addition, they provide insight into the sequence in time at which ions in the various charge states leave an evaporating droplet. ( $\mathrm{Am}$ Soc Mass Spectrom 1993, 4, 524-535)
\end{abstract}

$\mathrm{E}$ vaporation of charged droplets to produce free gas-phase ions from analyte species in solution was first proposed and investigated for mass spectrometry by Dole et al. [1] in the late 1960 s and early 1970s. They produced fine sprays of charged droplets by providing a strong clectrostatic ficld at the exit end of a small tube through which a dilute solution of analyte species was introduced into an atmosphere of nitrogen at atmospheric pressure. This socalled electrospray (ES) dispersion of a liquid had first been studied by Zeleny in the teens of this century [2]. Dole's idea was that cvaporation of solvent from such a droplet would increase the surface-charge density until it reached the Rayleigh limit at which the forces due to Coulombic repulsion and surface tension become comparable. The resulting instability would bring about a disruption of the parent droplet into a plurality of smaller offspring droplets that would continue to evaporate. A sequence of one or more such "Coulombic explosions" would ultimately give rise to droplets so small that each one would contain bul a single solute molecule. That molecule would become an ion by retaining some of the droplet charge as the last of the solvent vaporized. Although Dole et al.'s groundbreaking experiments indeed indicated that the charged droplets gave rise to solute ions, interpretation of their results remains somewhat equivocal because of problems in the method of mass analysis that they used. Even so, their pioneering investigations pointed the

Address reprint requests to John B. Fenn, Department of Chomical Engineering, Yale University, New Haven, CT 06520-2159. way to what has since become an extremely powerful analytical tool. However, in retrospect, they did not provide a very persuasive case for the charged residue model (CRM), which was the rationale for undertaking these investigations.

Despite Dole et al.'s provocative results, charged droplets were pretty much ignored as possible sources of ions for mass spectrometry until 1979, when Iribarne and Thomson [3] proposed their atmospheric pressure ion evaporation model (identified here as IEM) for ion formation. Two subsequent reports from the same group [4] refined the model and provided mass spectrometric evidence for its credibility. The IEM holds that a sequence of evaporation and Coulombic explosions, as in Dole et al.'s scenario, would lead to droplets with radii so small and charge densities so high that the resulting electrostatic field at a droplet surface would be sufficiently intense to lift solute ions into the ambient gas or vapor. The size regime in which such ion evaporation could occur might well be reached with fewer sequential Coulombic explosions than would be required to produce ultimate droplets containing only one solute molecule. In their experiments, Iribarne and Thomson generated charged droplets by pneumatic nebulization so that it is convenient, but not customary, to refer to their technique as aerospray (AS), in recognition of its use of aerodynamic forces to disperse the sample liquid. When the liquid being nebulized contains ions, the resulting droplets become charged during their formation as a result of statistical fluctuations in the distribution of anions and cations among them. Because charge must be conserved, it 
follows that nebulizing initially neutral liquid will produce both negatively and positively charged droplets in such a way that the sum of all charges over all droplets must equal zero. By suitable disposition of appropriately biased electrodes, one can select ions for sampling and analysis that are either positive or negative, even though both are produced as the droplets vaporize. Indeed, Thomson and Iribarne reported that a nearby induction electrode, at high potential relative to the nebulizing region, polarized the spray so that most or all of the charged entities, droplets, and the ions they produced, had the same polarity, opposite to that of the electrode [4]. Aeroelectrospray (AES) seems an apt name for this combination of aerodynamic atomization with electrical fields but, like AS, it is seldom seen.

Despite the promise of these early studies, the widespread use of charged droplets as practical ion sources had to wait until Vestal et al. [5] introduced thermospray (TS) ionization in 1980. That technique consists in passing sample solution through a narrowbore tube whose walls are hot enough to vaporize most of the solvent. The consequent expansion and acceleration of the vapor nebulizes the remaining liquid so that a jet of small droplets in superheated vapor emerges from the end of the tube. These investigators did not at first seem to consider that the ions they observed might have had their origin in charged droplets formed during nebulization of the liquid, as provided for by the IEM of Iribarne and Thomson. It was only later that TS ion formation became attributed in part to such droplets. Some investigators believe that ions in TS also result from gas-phase encounters of solute molecules with anions or cations from the volatile buffers used in liquid chromatography (LC) separations when TS is the interface to a mass spectrometric detector.

In the original Dole experiments, as noted earlier, dispersion of liquid into charged droplets was accomplished entirely by electrostatic work. As described, sample solution was introduced through a duct of small diameter maintained at a high voltage relative to the surroundings. The duct is frequently fashioned from hypodermic needle tubing and often referred to as the injection "needle." At voltages sufficiently high, the resulting field at the needle exit is strong enough to disperse the emerging liquid into a fine spray of charged droplets, all at the same polarity as the tube. Droplets produced in this way, purely by electrostatic forces, can be expected to have higher mass-to-charge ratios than those produced by TS, AS, or AES and thus provide higher analytical sensitivity. Clearly, the amount of electrostatic work done in dispersing a liquid is proportional to the amount of charge deposited on its surface. If some of that work is done by nonelectrical forces, then less charge will be deposited on the liquid surface. It seems reasonable to believe that the mass-to-charge ratio of a charged droplet determines the fraction of its solute molecules that can become free ions. Consequently, one might expect a lower ionization efficiency, or analytical sensitivity, when some of the work of dispersing a solution into droplets is performed by forces that are not electrostatic. In addition, because the selected ion current in electrospray mass spectrometry (ES/MS) depends directly on the concentration of analyte in the sample solution rather than on its flow rate, it has consistently been found that the lower the flow rate of sample solution, the smaller is the amount of analyte required for mass analysis. However, it is also well established that an annular flow of high-velocity gas around the needle exit can provide stable electrosprays at flow rates much larger than can be achieved without such a gas flow. Even a loss in sensitivity is sometimes a worthwhile price to pay to accommodate sample flow rates substantially higher than the maximum of $\mathbf{2 0}$ $\mu \mathrm{L} / \mathrm{min}$ or so at which a stable spray can be readily maintained by electrostatic forces alone. In their initial report on this "pneumatically assisted ES," Bruins et al. [6] proposed that it be called ion spray (IS), a somewhat misleading and unfortunate term that has since become a trademark for one of the suppliers of ES mass spectrometers and has led to some confusion.

The underlying process by which solute ions are formed from charged droplets would seem to be the same in all of these spray methods, no matter what they may be called or how they may be carried out. However, despite an explosive growth in their use over the past few years, especially in ES, there has been very little identification and elucidation of component mechanisms in the process by which solute species in a charged droplet are transformed into free gas-phase ions. With some vigorous exceptions [7], most investigators now seem to believe that the IEM of Iribarne and Thomson is more widely applicable than the CRM of Dole. Only in the experiments of Nohmi and Fenn [8] on ES/MS of polyethylene oxide oligomers having molecular weights $\left(M_{\mathrm{r}}\right)$ around $5,000,000$ is it fairly certain that the CRM was responsible for ion production. That said, there remain many questions, especially in the ES ionization of large molecules, that neither model has yet successfully answered. Among these questions are the following:

1. How can a single solute species give rise to a population of ions in which the number of charges per ion takes on all possible values between a minimum and a maximum?

2. What factors determine those maximum and minimum values?

3. How do small changes in solvent properties sometimes cause large changes in charge-state distribution?

4. In the ES mass spectrum for a large solute species, why do the peak heights have a bell-shaped distribution?

5. Why does the conformation of a solute species affect the charge-state distribution in its desorbed ions? 
This report addresses some of these questions and shows that the IEM seems able to provide credible answers when account is taken of some realities that have heretofore sometimes been ignored. These realities include the size, shape, orientation, and fugacity or escaping tendency of the solute species as well as the charge spacing and field at the droplet surface. As it turns out, the situation is so complex that a complete and quantitative analysis is still well out of reach. Even so, useful insight can be gleaned from the picture that seems to be emerging.

\section{Basic Assumptions and Nomenclature}

1. At the location in the spray where ions start evaporating, all ion-emitting droplets are assumed to have the same size, composition, and charge, having all been formed by the same sequence of events occurring at very nearly the same rate. Therefore, the composition of an ion sample withdrawn from the spray into the analyzer is identical to the time-averaged composition of all of the ions that have been produced from a single ion-emitting droplet up to the sampling point. The droplet undergoes no Coulombic explosions while it is emitting ions.

2. Each droplet is "well stirred" in the sense that its composition is uniform (but not constant) throughout the ion emission phase. At any instant it contains $N_{i}$ molecules of a single analyte species $i$. It also contains a number of charges $Q$ that represent excess anions or cations uniformly distributed on its surface. In the interests of simplicity, our considerations will relate to the case of droplets that are positively charged with an excess of $\mathrm{H}^{+}$cations, the adduct charges on ions desorbed from the droplets.

3 . The number of excess charges $Q$ on a droplet is large relative to the number of solute molecules in the droplet. For example, flash microphotographs taken under typical operating conditions for large molecules $(3 \mu \mathrm{L} / \mathrm{min}$ of $1 \mu \mathrm{mol}$ analyte in 1:1 methanol/water) show inilial droplet diameters of approximately 2.8 $\mu \mathrm{m}$. Simultaneous measurements of spray current indicate that each of those initial droplets has approximately 44,000 charges, corresponding to a $Q / N_{i}$ value of approximately 6. However, as has been shown in some elegant experiments by Taflin et al. [9], in typical Coulombic explosions the small offspring droplets carry off approximately $15 \%$ of the parent droplet charge but only approximately $2 \%$ of its mass, a gain of 7.5 in $Q / N_{i}$ for each succeeding generation of droplets. Thus, after three such explosions, the $Q / N_{i}$ value is approximately 253.5! Still not known is how many such explosions take place betore offspring droplets start emitting ions, but two or three would seem to be reasonable guesses. Further evidence that $Q / N_{i}$ remains high during ion emission can be inferred from the observation that for large ions there is a wide range of $z$, the number of charges per ion as determined from the measured mass spectra. Justification for this inference will be deferred until later (see Discussion).

4. The excess charges $Q$ are distributed over the surface of the droplet in a pattern such that they are equidistant from one another because with such a spatial distribution the electrostatic potential energy is at a minimum. It is appropriate to examine the magnitude of the electrostatic potential energies involved in holding a surface charge to a particular position. It will be assumed in these and later considerations that the distance between charges on a desorbed ES ion is about the same as the distance between the charges on the surface when that ion desorbed. Thus, for example, in the case of desorbed ES ions of poly(ethylene glycol) with $M_{\mathrm{r}}=8000$, the distance between charges $\left(\mathrm{Na}^{+}\right)$ is approximately $40 \AA$. A rough estimate, based on a pairwise sum of Coulombic interactions between one charge and its nearest four neighboring charges along a line in either direction, indicates that when the charges are $40 \AA$ apart, a displacement of $5 \AA$ corresponds to a potential energy change equivalent to approximately $1.5 k T$ at $325 \mathrm{~K}$, the approximate drying gas temperature where the droplets are evaporating. If one includes the repulsion from more distant ions along that line, and from ions not on it, but with components of electrostatic force parallel to it, the potential energy "well" becomes even deeper. Thus, for a surface charge to bind to a molecule, the binding site on that molecule must be within approximately 5 $\AA$ of a charge.

It would be more accurate to characterize the equilibrium distribution of surface charges as corresponding to a minimum in the total chemical potential $\mu$ of those charges. Thus, in principle, one should take into account the contributions to $\mu$ arising from temperature, pressure, and concentration of the charged species on the surface as well as from their electrostatic potential energy. However, the temperature and pressure are uniform, and the distribution of any surface species would also be uniform in the absence of any charging. Thereforc, the equidistant spacing of charged species that corresponds to minimization of electrostatic potential energy in effect simply reinforces what would occur if the species were not charged. Moreover, as shown above, the electrostatic energy "well," in which each charge finds itself at equidistant spacing on a droplet, is relatively deep compared with $k T$. In view of these considerations, it seems safe to assume that the electrostatic component of the chemical potential governs the location of a charged species on the surface of a charged droplet.

Attachment of a surface charge to a site on a solute molecule might seem to offer the possibility of displacing the charge from its position of minimum electrostatic energy. For example, if a surface proton were attached to a basic site on a protein molecule, movement of that molecule might then drag it away from its 
minimum energy position. However, the only possible motion of the molecule is Brownian in nature, with an energy on the order of $k T$, so that in fact the amplitude of any proton displacement would be smaller if it were attached to a massive molecule than if it were unattached. Rather, the protor would serve to anchor the molecule, or at least its attachment site, to the minimum energy position. Thus, once a single basic site on a protein molecule becomes attached to a surface proton, any translational displacement of the molecule would be limited to Brownian oscillations around a fixed point. In addition, of course, internal bending, vibration, and rotation could take place, also with amplitudes constrained by $k T$. If the proton spacing on the droplet surface is small enough, such Brownian thrashing about of a large anchored molecule could bring one or more of its other basic sites to the vicinity of one or more of the other surface charges, which could then become attached to the molecule. Each such attachment would anchor another segment of the molecule to a charge site. Clearly, the total number of charges that could become attached to the molecule by this process would depend on the size, shape, and conformation of the molecule, along with the location of its charge sites. To be remembered is that the binding of a proton to a basic site, or indeed the binding of any charge to a polar group or atom on any molecule, is due to ion-dipole attractive forces that have a much shorter range than the Coulombic repulsion forces between separated charges. Consequently, a prospective attachment site on a large molecule would have to get fairly close to a surface proton for attachment to occur. In other words, binding to a molecule does not enable a charge to move laterally very far from its minimum energy location.

5. In accord with Iribarne and Thomson, we assume that the evaporation or desorption of analyte ions from the droplet can be treated as an activated process describable by a rate equation in Arrhenius form:

$$
\dot{N}_{i z}=A n_{i} S \exp \left(-\Delta G_{i z} / R T\right)
$$

in which $\dot{N}_{i z}$ is the flux from the droplet surface of ions that comprise a molecule of analyte species $i$ to which $z$ charges are attached. The concentration $n_{i}$ of species $i$ in the bulk liquid of the droplet is given by $N_{i} / V$, where $V$ is the droplet volume, $S$ the surface area of the droplet, and $A$ a constant that includes steric factors as well as the proportionality constant that relates the bulk concentration $n_{i}$ to its number density in the surface layer. The activation energy $\Delta_{\text {Giz }}$ is the difference in Gibbs free energy of an ion of species $i$ with $z$ charges when it is on the surface of the droplet and when it is far enough away from that surface so that solvation forces are negligible. It is then "free" in the sense that its motion will be determined entirely by the interaction between an acceleration force due to the electrostatic field and a drag force due to the viscosity of the ambient gas. We assume that the energy difference between a solute molecule in the bulk of the droplet and on its surface is negligible so it can move freely by diffusion within the droplet. Thus, any net motion of solute can result only from concentration gradients. As noted earlier, we also assume that this diffusion is so rapid that the bulk concentration of the solute remains uniform but not constant during ion emission. Clearly, the flux of charge, or current, carried by departing ions with $z$ charges is simply the product of eq 1 and $z$.

6. Perhaps the most important term is $\Delta_{G i z}$, the activation energy barrier that an ion must surmount to escape the droplet. We assume that this quantity can be represented by

$$
\Delta G_{i z}=\Delta G_{i z}^{o}-z f \Delta r^{*}
$$

where $\Delta G_{i z}^{\circ}$ is the work required to remove an ion of species $i$ with $z$ charges from the surface of the droplet. The term $z f \Delta r^{*}$ is the portion of that work that can be contributed by the field acting on the charge through the distance $\Delta r^{*}$ which the ion must traverse to be "free" of the droplet in the sense of being over the activation-energy barrier. If an ion is to leave the droplet, the difference between $\Delta G_{i_{z}}^{\circ}$ and $z f \Delta r^{*}$ must be provided by the thermal energy of the ion at the droplet temperature $T$ and is represented by $R T$. We make the simplifying assumption that $\Delta r^{*}$ does not depend on $z$ and so is the same for all ions of species $i$. In addition, we make the somewhat more suspect assumption that $\Delta G_{i z}^{\circ}$ is also independent of $z$. A free charge such as $\mathrm{H}^{+}$can have a strong attractive interaction with molecules of a polar solvent. The result is an atmosphere of solvent molecules that cluster around the charge. The charges on a large ion are far enough apart to interact independently with ambient solvent molecules. Thus, the number of solvent molecules clustered around the ion should be proportional to $z$. These solvent molecules probably stay with the desorbing ion, increasing its effective surface area. Therefore, the increase its total surface area of the dropletion system increases when the ion desorbs. A significant fraction of $\Delta G_{i z}^{\circ}$ is "surface work, given by the product of surface tension and the increase in surface area on desorption. As we will see, to increase an ion's $z$ requires an increase in droplet-surface charge density that also decreases effective surface tension, (i.e., the surface tension characteristic of the droplet liquid less the repulsion clue to droplet surface charge). In sum, a significant portion of $\Delta G_{i z}^{\circ}$ is represented by the work required to increase the surface area of the ion-droplet system as the ion desorbs. That work is represented by $\sigma \Delta S$, where $\sigma$ is the effective surface tension and $\Delta S$ the increase in system area on desorption. As increasing surface-charge density increases $z$ for a desorbing ion, the surface area of that ion also increases, thereby increasing the $\Delta S$ of the system caused by desorption. But increasing surface-charge density also increases Coulombic repulsion and de- 
creases the effective surface tension $\sigma$ of the droplet liquid. Thus, accompanying an increase in $z$ for a desorbing ion is a decrease in $\sigma$ and an increase in $\Delta S$. Clearly, these two corollaries of an increasing $z$ have opposing effects on the work associated with the increase in system area (i.e., $\sigma \Delta S$ ). We simply assume that they offset each other so that $\Delta G_{i z}^{\circ}$ changes very little with $z$.

7. If $V$ and $S$ are expressed in terms of the droplet radius $r$, and the field $f$ is written as $Q / 4 \pi \epsilon_{0} r^{2}$, where $\epsilon_{0}$ is the permittivity of the ambient gas, eq 1 then becomes

$$
\dot{N}_{i z}=B(N i / r) \exp \left(C z Q / r^{2}\right)
$$

where $B=3 A \exp \left(-\Delta G_{i z}^{\circ} / R T\right)$ and $C=\Delta r^{*} /$ $4 \pi \epsilon_{\mathrm{a}} K T$.

\section{Discussion}

In principle, if the injected solution contained only a single species $i$, then integration of eq 3 for a droplet decreasing in size from $r_{1}$ (when ions of species $i$ having $z$ charges start desorbing) to $r_{2}$ (at the point where ions are sampled) would give a relative value for the number of ions with $z$ charges in the sample of ions from the total population at that point. Summing the results for similar integrations, one for each value of $z_{x}$ would provide the composition of the population (i.e., the relative numbers of ions of each species $i$ with $z$ charges). To determine the composition of the total population if more than one solute species were present in the droplet, one would have to carry out a similar integration for each species. Unfortunately, the information required for such integrations is not yet available. We still have no idea of the actual values, initial or final, for most of the terms in eq 3 , whether they are variable or constant. Even if we knew the initial and final values, we would also need to know their dependence on time (i.e., the evaporation path in $r-t$ space). However, we can gain some insight as to what goes on by examining the expected response of eq 3 and romparing that response to experimental observations when the following variables are changed.

1. Initial Analyte Concentration $n_{10}$. We note first that when $z$ is unity, the value of $N_{i}$ on the right-hand side of eq 3 is directly proportional to $n_{i}$, and none of the other terms have any dependence on $n_{j}$. If, for example, $n_{i 0}$, the initial value of $n_{i}$, is doubled, then the value of $n_{i}$ at all stages in the integration will also be doubled. In other words, the integral of the left-hand side will be proportional to $n_{i 0}$. Experiments with many different species have shown such a linear dependence of signal over four or more decades of change in $n_{i 0}$. Departure from linearity in a plot of signal against $n_{i 0}$ usually occurs as a fairly abrupt decrease in slope when when $n_{i 0}$ reaches a sufficiently high value. This decrease is attributable to $n_{i}$ having reached its saturation value during droplet evaporation so that further luss of solvent results in precipitation of $i$ rather than in any further increase in its concentration in solution. Figure 1 shows data taken in our laboratory by Takashi Nohmi reflecting this behavior for three tetraalkyl ammonium halides that produce singly charged ions. Such linearity with $n_{i 0}$ may not be found in the case of ions with more than one charge because the exponential term in eq 3 is $z$ dependent.

2. Dependence on $\Delta G_{i z}^{\circ}$. It is difficult to provide a quantitatively controlled variation of this variable, but some insight is given by Figure 1, which shows that at all concentrations the selected ion current increases with increasing chain length of the alkyl groups on the quarternary nitrogen. This dependence is set forth more explicitly in Figure 2, where the ordinate values indicate that the mass spectrometric response (selected ion current) increases by almost fifteen-fold as the number of carbon atoms per alkyl chain goes from one to four. Such an increase in chain length is accompanied by an increase in solvophobicity for the molecules so that less work should be required to remove them from the droplet as the chains get longer. In other words, $\Delta G_{i z}^{\circ}$ should decrease so that according to $e q 3$, ion current should increase. The results in Figure 2 bear out this

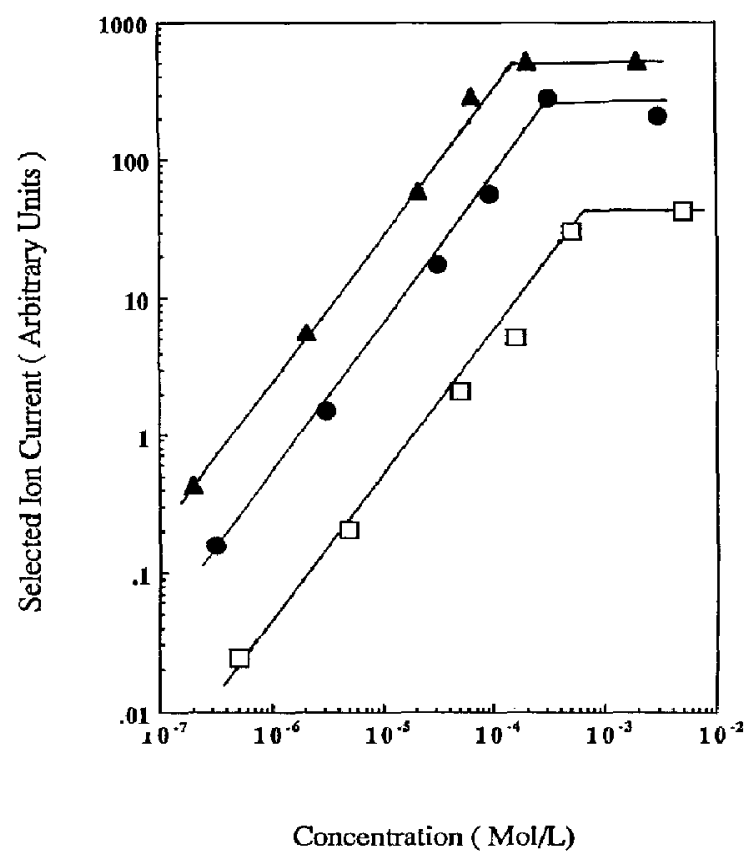

Figure 1. Dependence of mass spectrometry signal (selected ion current) on initial solute concentration $n_{i 0}$ for tetramethyl ( $\square$ ). tetrabutyl (O), and tetraheptyl ( $\Delta$ ) ammonium halides. The coordinate scales are logarithmic, but the lines have slopes of unity, so signal is directly proportional to $n_{i 0}$. The concentration units along the abscissa are in moles per liter. The ordinate values are selected ion currents in arbitrary units. 


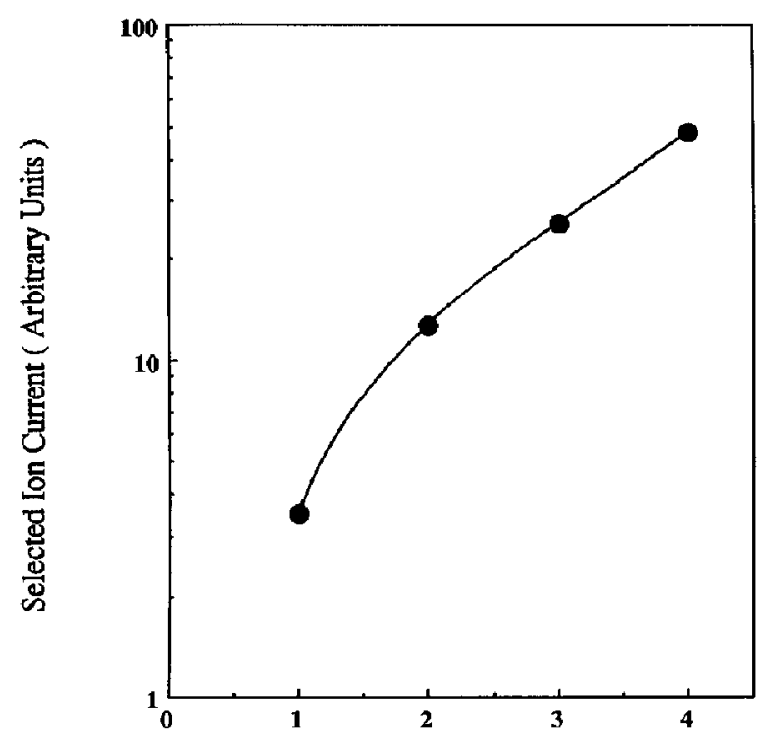

$C$ atoms in alkyl chains

Figure 2. Dependence of mass spectrometry signal on hydrocarbon chain length for tetraalkyl ammonium halides from methyl through butyl. The ordinate values are mass spectrometry signals (selected ion currents) in arbitrary units. The abscissa values are the number of carbon atoms in the alkyl groups. As the hydrocarbon chain lengthens, $\Delta C_{i z}^{\circ}$ for the solute decreases, and desorption rate increases as eq 3 predicts. All solutions were 10 $\mu \mathrm{mol} / \mathrm{L}$ in analyte. It is noteworthy that with a $10-\mu \mathrm{mol} / \mathrm{L}$ solution of ammonium bromide, in which the number of carbon atoms per side change is zero, the selected ion current for ammonium ion was at least $\mathbf{5 0}$ times less than for the tetramethyl salt, reflecting the high value of desorption free energy for that small, very hydrophilic ion.

expectation. Unfortunately, the exact relation between $\Delta G_{i z}^{\circ}$ and chain length is not known, so that the variation of signal cannot be quantitatively described a priori. It is interesting to note that as chain length increases still further, the signal diminishes because lower solubility decreases $n_{i}$ in the desorption regime. Indeed, it is well known that compounds with very long alkyl chains, like stearic acid or cetyl alcohol, are so solvophobic (i.e., their $\Delta G_{i z}^{\circ}$ values are so low) that dispersed in water with a volatile cosolvent they spontaneously "leave" the solution to form an unwetted film on its surface, with only the hydrophilic ends of the molecule bound to the substrate liquid. Such films are known to be an effective barrier to evaporation. Indeed, Iribarne, Dziedzic, and Thomson [4] found in their AES experiments that the presence of decyl alcohol completely inhibits ion production, probably because it forms a surface film that prevents droplet evaporation.

3. Charqe-State (z) Distribution. Equation 3 shows that desorption rate depends exponentially on $z$, but it gives no clue as to what determines charge state. Some "explanations" have been offered for the numbers of charges per ion that are observed. For example, in their first report on $\mathrm{ES} / \mathrm{MS}$ of proteins, Covey et al. [10] suggested that the maximum number of charges on an $\mathrm{ES}$ ion of a protein or peptide is equal to the number of basic residues (i.e., amino acids such as arginine and lysine) that it incorporates. That inference is plausible but somewhat incomplete. A more correct and more general embodiment of its essential premise would relate the maximum number of charges on an ES ion to the total number of polar groups or sites to which adduct charges might attach, subject to the constraint that after the ion has left the droplet, the bonding energy of any one charge to any one site must equal or exceed the electrostatic repulsion energy due to Coulombic repulsion by other charges on the ion $[8,11]$. Even in this more complete form, the proposition does not account for the wide variation in the numbers of charges that are found on ES ions of large molecules.

Smith and co-workers [12] found that ES ions of a protein with its disulfide bond cross-links intact had fewer charges than when ionization occurred after the cross-links had been chemically ruptured. In an extension of the one charge per basic residue notion, they suggested that in the more compact configuration (cross-links intact), some of the possible charge sites (basic residues) were not accessible to the charges. A similar rationale was invoked by Chait and co-workers [13] to explain the smaller number of protons per ion of cytochrome $c$ when it was in a more natural conformation than when it was denatured and spread out. These arguments do not ring entirely true. Protons are pretty small, so the configuration would have to be pretty dense to exclude them. Moreover, when a protein folds, the hydrophobic hydrocarbon segments usually huddle together in the core of the molecule, leaving the hydrophilic polar groups (prospective charge sites) exposed to the solution.

More recently, Siu and co-workers [14] noted that the distributions of charge states in ES ions are similar to those they calculated (from the $\mathrm{p} K_{\mathrm{a}}$ values for the amino groups on the molecule) for the ions in the bulk solution. Indeed, they even suggested that charging and evaporation of the ES droplet is not required for production of the ions because they are already present in the initial solution sample. That notion is defensible if the term "production" refers to the formation of ionic species in a solution that is neutral overall. It is much less tenable if it refers to the formation of free ions in the bath gas. The calculations of Siu and coworkers relate to ion populations in neutral bulk solution. Desorption of ions from neutral droplets has never been observed, even when such ions are known to be present in the solution. Indeed, desorption of a positive ion from a neutral droplet would leave that droplet with a net negative charge that would attract the departing positive ion right back to the droplet. In the perspective of this report it is the situation at the surface of a charged droplet that determines the nature 
and amount of charge on a departing ion. When the $\mathrm{pH}$ is low enough in the bulk solution of a positively charged droplet, there will be on the surface an excess of $\mathrm{H}^{+}$ions, perhaps with varying degrees of solvation. If, for example, the neutral bulk solution within the droplet also contains protein molecules, each proton attached to a basic residue on one of those molecules in the bulk will have a nearby anion as its "shadow," so that overall neutrality is maintained. When such an "ion pair" approaches one of the surface protons, the nearby anion will remain "neutralized," either by the surface proton or by the proton attached to the basic residue, leaving in either case a net positive charge of one proton associated with that basic residue at that surface location. On the other hand, an unprotonated basic residue (amino group) that approaches a surface proton closely enough can attach that proton and thus become a charged group on the protein at that location. To be sure, as in the case of protonated groups in the bulk solution, there are anions around that could in principle associate with that surface proton, whether or not it is attached to a molecule, but only by "releasing" another positive charge. Thus, there will always be a net positive charge at that location. In sum, one or more of the net excess charges on the surface of a droplet can always attach to a parent molecule, providing it with a net charge that by interaction with the surface field will help lift that molecule from the surface when the conditions are right for desorption. As discussed in more detail later, when the surface charges are close enough together so that several can be embraced by a single protein molecule, that molecule will then have several net charges that can help lift it from the surface. The point is that the number of protons found on a desorbing protein ion is determined by the number density of net protons on the droplet surface, not by the number of the protein's basic groups, which on average are protonated in the neutral bulk solution. This perspective is elaborated in what follows to construct a new scenario that seems able to provide at least a qualitative explanation for most of the features of charge-state distributions that have been found in ES ions.

It was argued earlier in this discussion that the charges on an ES droplet are distributed over its surface with equidistant spacing and that they are locked into this pattern by the forces of Coulombic repulsion. As the droplet evaporates, the charges move closer together, thus increasing the surface-charge density and the field normal to the droplet surface. A basic premise of this new scenario is that the charges on a desorbing ion cannot be any closer together than they were on the droplet when the ion desorbed. Thus, the number of charges that are attached to an analyte molecule when it leaves the surface as an ion is the number of charges that it can span at the spacing that obtains on that surface.

The situation is illustrated in Figure 3, which shows projections of the surface of the same droplet at three different sizes, corresponding to different stages of

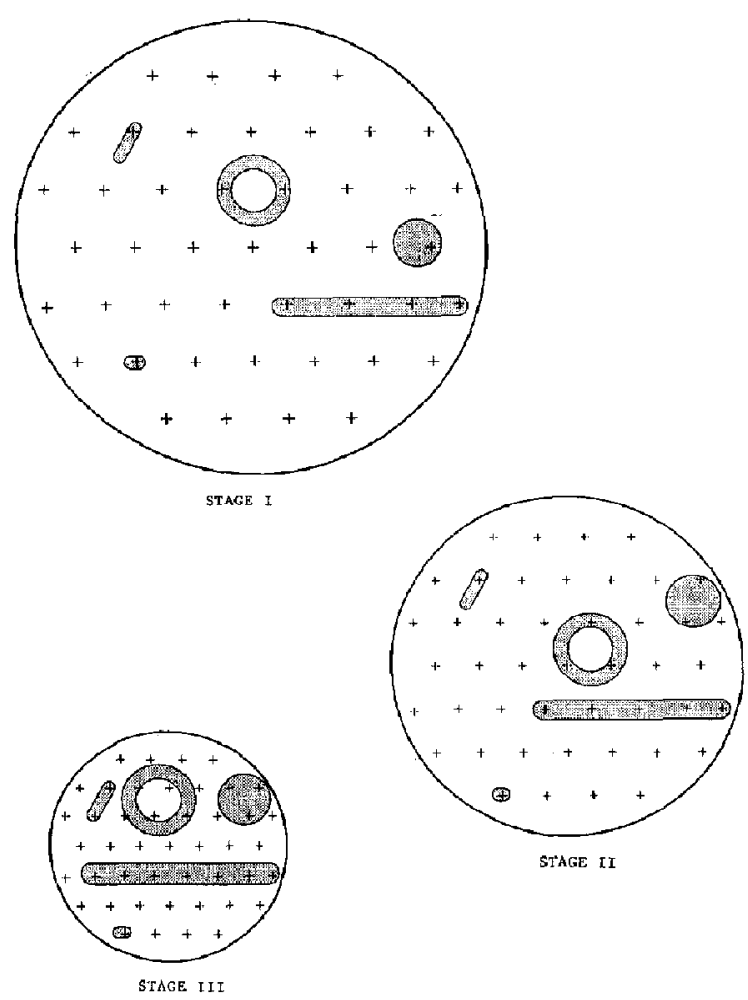

Figure 3. A two-dimensional "map" showing schematic projections of cross sections for five molecules of different sizes and shapes on the surface of a droplet at three stages of evaporation (I, II, and III). As droplet size derreases, the molecule size and total number of droplet charges remain constant, but the charge spacing decreases. In general, the higher the surface charge density, the greater is the number of charges within reach of binding sites on a molecule.

evaporation that are referred to in order of increasing time (decreasing size) as I, II, and III. As the dropiet size decreases by vaporization of solvent, the distance hetween the equally spared positive charges decreases. Also disposed on the surface are heiroglyphs representing five molecules differing in size and/or shape. The shaded area enclosed in the lines forming these sketches is a projection representing in each case the area of the surface that is within the molecule's "sphere of influence." The smallest molecule will be referred to as the "dot" and the next largest as the "oval." The three largest sketches all encompass the same surface area within their defining lines. They represent the same assemblage of atoms in three different configurations and are referred to, respectively, as the "bar," the "ring," and the "disk." The underlying assumption in what follows is that the maximum number of charges that can lie within the perimeter of the molecule (i.e., covered by its shaded area) is the maximum number that can attach to that molecule when it desorbs as an ion from the surface of the droplet. It would be more accurate to say that the only charges that can attach to the molecule are those within reach 
of prospective attachment sites on the molecule; however, to make the essential point as simply as possible, we assume that a charge can attach at any location within the molecule's projected area that is shaded on the drawing.

Clearly, at evaporation stage $I$, the dot and the oval can embrace only one charge. If the surface field for the indicated charge density is sufficiently strong, the dot and the oval will then evaporate as singly charged ions at rates defined by eq 3 . As evaporation brings the droplet to stage II, the dot and the oval can still attach only one charge, but they will desorb at a faster rate because the surface field is higher because of the greater surface-charge density. At stage III, desorbing dot ions will still have only one charge, but the oval molecule can embrace two charges so that it can give rise to both singly and doubly charged ions. In accord with eq 3 , the specific desorption rate will be higher for the doubly charged ions than for those with a single charge. However, the actual flux might be lower because the surface concentration is much lower for doubly charged ions than for singly charged ions. The reason is that there are more combinations of location and orientation that would enable a molecule to encounter one charge than would enable it to span two charges. As evaporation continues, the charges will become so close together that oval molecules would find it increasingly difficult to avoid simultaneous contact with two or more ions. At that point, the desorption flux would become much smaller for ions with one charge than for ions with two. However, even at very high surface-charge densities, the number of charges that an ion can carry is limited by its size and the bonding energy between a charge and its site [11].

Equation 3 shows that two other factors are also involved in determining the flux and charge state for desorbing ions of a particular solute species at any particular evaporation stage of the host droplet. Those factors are the surface concentration of that species and its desorption free energy, discussed in the preceding sections. Thus, it becontes apparent, even for relatively small and simple species, that many variables play a role in determining the abundance and charge state of the ions that are found experimentally. Despite these complications, it may be worthwhile to consider in some defail an experiment of historical interest. The first accurately analyzed ES ions with $M_{\mathrm{r}}$ values above 400 were of gramicidin S (gmc) and cyclosporin A (csp), two cyclic peptides of almost the same size, with $M_{\mathrm{r}}$ values of 1141.5 and 1202 , respectively. An intriguing feature of their mass spectra was that at all concentrations, the observed abundance ratio $\mathrm{M}^{2+} / \mathrm{M}^{\prime}$ was much higher for gmc than for csp. 'Ihis ratio approached unity for gmc at low $n_{i 0}(<0.01 \mathrm{~g} / \mathrm{L})$ and decreased with increasing $n_{i l}$ but never became as small as for esp, which produced a ratio of order 0.02 at an $n_{i 0}$ of $0.1 \mathrm{~g} / \mathrm{L}$. In the perspective of eq 3 and Figure 3 (where these two molecules can be represented by the oval), this behavior becomes understandable. Solubility and liquid chromatographic re- tention times show that in the solvent used for ES/MS measurement (1:1 methanol/water), csp is much more solvophobic than gmc, indicating a smaller value of $\Delta G_{i z}^{\circ}$ (greater fugacity) for the former. Therefore, the singly charged csp ions can desorb substantially faster at lower fields and thus at lower charge densities. Hence, the flux of its singly charged ions starts sooner and becomes greater at earlier stages of evaporation. This behavior can be readily inferred from Figure 3, in which we can relate the oval molecule to these two species. In stages I and II, the oval cannot span two charges, so it can leave only with a single charge at a rate determined by the droplet charge density (field) and $\Delta G_{i z}^{o}$. Therefore, csp provides a much higher flux of singly charged ions. At evaporation stage III and beyond, the oval can span two charges, so that doubly charged ions can be desorbed. Then, too, as in the early stages when all of the ions were singly charged, $\operatorname{csp}^{2+}$ would lift off faster than $g m c^{2}$, unless the supply of csp molecules had been so depleted by losses as $\operatorname{csp}^{+}$that not enough remained to produce many $\operatorname{csp}^{2+}$ ions. If the initial value of $n_{i 0}$ were very high, then the rates of desorption for both $\mathrm{gmc}^{+}$and $\mathrm{csp}^{+}$could be high enough to carry off most of the droplet's charge, so that very few doubly charged ions would show up in the spectra for the sampled population. Indeed, the experiments did show that as $n_{\text {i0 }}$ increased much above $0.1 \mathrm{~g} / \mathrm{L}$, even most of the gmc ions were singly charged. Conversely, at very low $n_{i 0}$. most of the csp ions were doubly charged. This relatively simple case, involving only singly and doubly charged ions, was treated in some detail to illustrate and elucidate the interplay of the various factors that affect ES ion production according to the model as charactcrized by $\mathrm{eq} 3$. That model seems to provide an explanation for observed ES behavior of these two species that is plausible and self-consistent even though it is more qualitative than quantitative. We now take a much less detailed look at what can happen with larger species.

First we consider the bar molecule in Figure 3. At stage $I$ in the droplet vaporization it cannot possibly capture more than four charges. If the $\Delta G_{i z}^{\circ}$ is sufficiently small so that bar ${ }^{4+}$ can desorb al the field associated with the stage 1 surface-charge density, then bar $^{4+}$ should appear in the measured spectrum. Indeed, if $\Delta G_{i z}^{\circ}$ is small enough, then bar ${ }^{3+}$ or perliaps even $\mathrm{bar}^{2+}$ and $\mathrm{bar}^{+}$might also appear, although their abundances would be much lower for the same $n_{i}$. However, if $\Delta G_{i z}^{\circ}$ were to be so small that ions with lower charges could readily desorb at the lower charge densities encountered in carlier stages of the evaporation, then most of the bars might have left the droplet as ions in those lower charge states so that bar ${ }^{4+}$ might be present only in very small amounts. If $n_{i}$ were to be high enough to produce bar ${ }^{4+}$ at stage I, then in fact it might have been high enough in the previous stages so that the flux of ions in lower charge states during those preceding stages could have been sufficient to deplete the original droplet charge so 
much that the charge density of stage I might not be achievable. In that case, no $\mathrm{bar}^{4+}$ ions would be observed.

In stages II and III of Figure 3, the charge densities are high enough so that the bar molecule can span five and six charges, respectively. Consequently, $b^{2} r^{51}$ and bar $^{6+}$ should show up in the spectra, if that possibility had not been eliminated by behavior in the preceding stages. That is to say, behavior to be expected in stages II and III would follow a script exactly analogous to that for behavior in stage I, mutatis mutandis. It would be subject to the same kinds of constraints imposed by what had taken place in previous stages, as discussed in the preceding paragraph for stage I. It will now be shown that implicit in this script are the reasons for the bell-shaped distribution of charge-state populations that are characteristic of ES mass spectra. (Examples of such spectra are shown in Figure 4).

As an initially formed droplet starts shrinking by evaporation, its surface-charge density and the associated field both increase. Solute molecules will begin to desorb as ions at a measureable rate when the distance between charges on the droplet surface decreases to the point where the molecule can span $z$ of them if the field at that charge spacing in combination with the value of $\Delta G_{i,}^{\circ}$ can produce "lift-off." In that circumstance, molecules with $z$ charges attached will start desorbing at a rate that continues to increase in accordance with eq 3 , as evaporation continues to decrease the droplet radius. The charge spacing also decreases with evaporation until the solute molecule can span $z+1$ charges to make possible the formation of ions with $z+1$ charges. Each one of those $z+1$ ions will have a shorter lifetime on the surface than the $z$ ions because of the greater lift. As the charge spacing continues to decrease, the population of $z+1$ ions will also increase because of a concomitant increase in the number of combinations of location and orientation that will allow the molecule to span $z+1$ charges. Ultimately, the rate of desorption of ions with $z+1$ charges will exceed the rate for $z$ ions. The reason is that at sufficiently high surface-charge densities, almost every molecule will be in contact with $z+1$ charges, so the shorter residence time on the surface of $z+1$ ions will result in their preponderance in the flux from the surface.

As the droplet continues to shrink, the factors described will lead to a preponderance of $z+2$ ions in the flux from the surface. Sooner or later, however, the flux of all ions from the surface must start to decrease as the supplies of charge and/or solute species become depleted. The supply of charge on any droplet is ultimately limited by the Coulombic explosion phe nomenon and the supply of solute by saturation effects. Consequently, the flux of ions in the highest charge states must decrease along with the time during which the highest fluxes can be maintained. Therefore, above some critical number of charges, the relative abundance in the spray of ions with charge states above that critical number must decrease and finally vanish as the number of charges per ions increases. Also to be remembered, as noted earlier, is the intrinsic upper limit on the number of clarges that a molecule can carry because of competition between electrostatic repulsion and bonding energy [11].

4. Role of Molecular Conformation. As noted above, the ring and disk in Figure 3 represent different conformations of the same aggregation of the same building blocks as the bar, so they show the same projected area. In other words, the folding and compacting have taken place in two dimensions. For real molecules, the folding of the bar in three dimensions would result in smaller projected areas than are represented by the ring and the disk. 'I'he point to be made is that the greater the extent of compaction, the smaller is the number of charges that the molecule can span. Thus, in stages I, II, and III, the disk can embrace, respectively one, two, and three charges, the ring two, three, and four charges, and the bar four, five, and seven charges. If ions corresponding to these charge states are to appear in the sampled population, then the fields associated with the corresponding charge densities must be sufficient to provide lift-off in a sufficiently short time. Consequently, one can ask whether two charges in the field of state I would provide enough lift in the associated field to produce a measureable flux of disk ${ }^{2+}$ ions. If not, then disk $^{2+}$ ions would not appear until a later stage, when the charge spacing had decreased enough to provide the combination of field strength and number of accessible charges to bring about a measurable flux of ions with two charges.

Although not so explicitly set forth in the previous discussion, it is indeed the combination of field strength and required lift $\left(\Delta G_{i z}^{\circ}\right)$ that determines the minimum number of charges that any molecule must capture if it is to become part of the population of desorbed ions that can be obtained with a particular species. Thus, bar ions might be produced at an earlier stage of evaporation than disk ions. On the other hand, in its compact configuration, a molecule has a smaller surface area in contact with the solution than when it is unfolded. Consequently, less work may be required to remove it from the droplet, so that it could lift off with fewer charges than when it is unfolded. In the case of bar molecules, the script that we have been describing would insist that the greater the number of charges on the desorbed ions, the later would be the stage in the evaporation process at which they were produced. However, as outlined above, molecules of the same composition but more compact configuration might have to wait until later stages to be produced, even though they show up with fewer charges, simply because they cannot reach the charges they need except at charge densities attained only at later stages of droplet evaporation.

5. Effects Due to Droplet Evaporation Rafe. The discussion of eq 3 pointed out that integrating it would not be possible unless the dependence of droplet radius on 

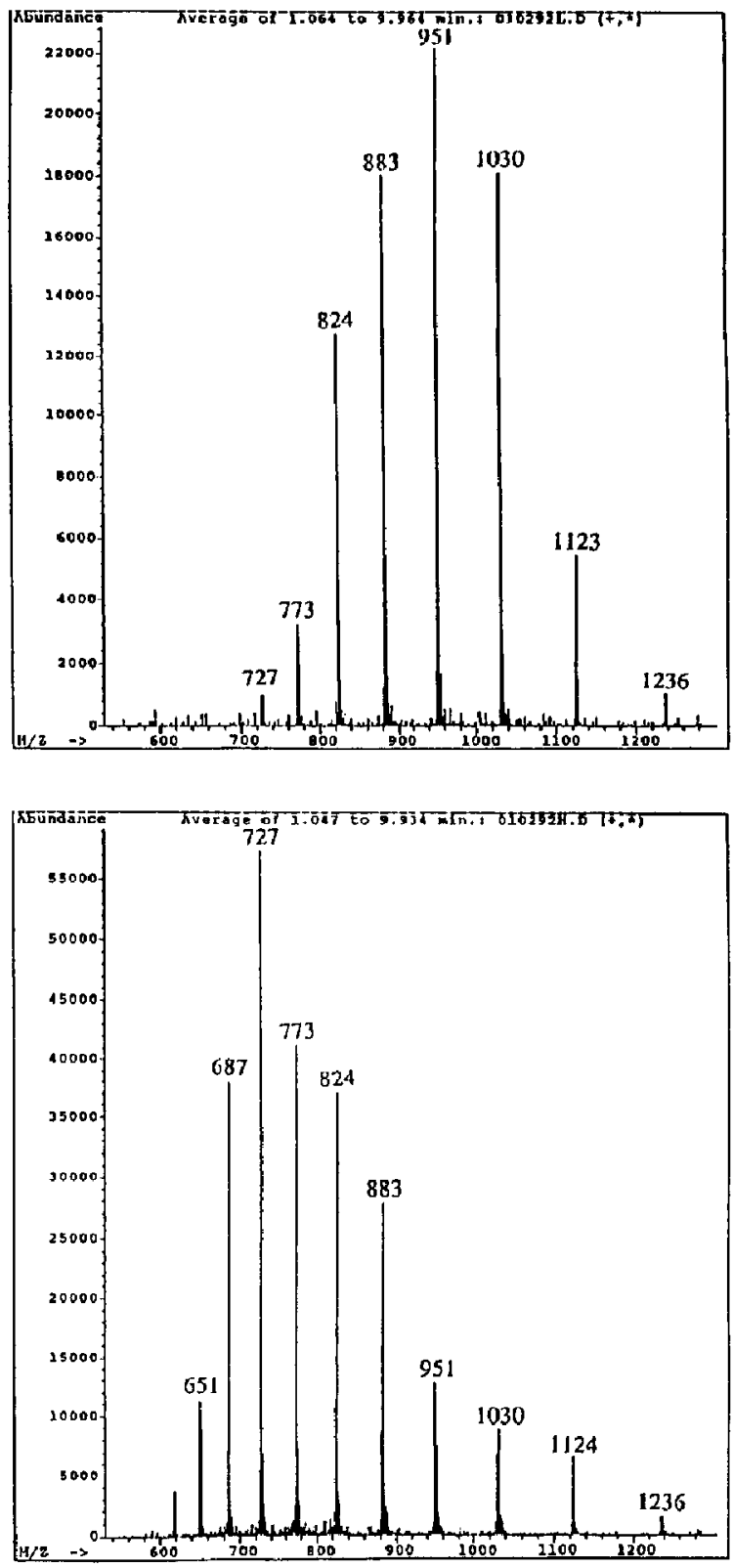

Figure 4. The effect of droplet evaporation rate on charge-state distribution in $\mathrm{ES}$ ions of cytochrome $c$ in methanol /water (1:1) at a concentration of $0.8 \mu \mathrm{mol} / \mathrm{L}$. The flow rate of drying gas in the lower spectrum is about six times that in the upper spectrum. The flow rate for cooling gas was the same for both spectra. There is a clear shift to higher charge states (lower mass-to-charge ratio values) at the higher flow of drying gas for which the evaporation rate is faster.

time could be specified. In light of the immediately preceding comments it should be clear that the shape of the charge-state distribution should indeed depend on the time history of droplet radius. For example, we concluded that in general the ions desorbed at the low surface-charge densities that occur in the early stages of evaporation lift off with fewer charges than those that desorb at later stages when the surface-charge density is high. Thus, if the evaporation rate is very slow, the droplet would spend a relatively long time in the regime of low surface-charge density. Then there could be a relatively large contribution of ions in low-charge states to the total population, even though such ions desorb very slowly. Because the supply of both charge and analyte molecules is limited, those low-charge ions are formed at the expense of those 
with higher charge that desorb at a rapid rate but carunot start until the droplets become small enough. In other words, if the picture that has been painted here is a reasonable reflection of reality, rapid evaporation of droplets should lead to charge distributions biased to the high-charge states. Conversely, slow evaporation rates should increase the relative abundance of ions with low-charge states. In the course of developing an ultrasonically assisted ES source, Shida Shen at Analytica of Branford obtained some results that support this speculation on the effects of evaporation rate.

That source incorporates a flow of cooling gas around the injection needle to dissipate some of the heat generated by the ultrasonic vibration. The veloc ity of that flow is too low to affect the nebulization so that varying its velocity over a modest range does not influence the size or charge of the droplets produced. Moreover, that flow is concurrent with the drift of charged droplets and desorbed ions toward the inlet of the glass capillary that passes some of the ion-bearing bath gas into the vacuum system containing the mass analyzer. Flowing in the opposite direction, countercurrent to the drift of charged droplets and desorbed ions, is the heated bath gas that is introduced in an annular flow around the glass capillary entrance. The relative rates of flow for the countercurrent bath gas and the concurrent cooling gas can be adjusted independently. If the bath gas flow is fixed, increasing the flow of cooling gas will tend to slow down the evapo ration rate of the droplets for two reasons. The velocity of the droplets relative to the gas decreases, thus slowing down transport processes, and the droplets spend more time in the cooling gas, which is moist because it contains vapor that has evaporated from the droplets. Another consequence is that the droplets spend less time on their way to the capillary because the velocity of the cooling gas is added to the migration velocity due to the field. If evaporation is not yet complete when the droplets reach the plane of the capillary aperture, the ions entering the capillary are likely to be more representative of what happens earlier rather than later in the evaporation process. All of these effects act in the direction of increasing the relative amounts of ions in the sampling flow that desorb in the early stages of the droplet vaporization. Hence, one would expect to find the charge distribution shifted so that more of the ions have fewer cluarges. Conversely, if the cooling gas flow rate is fixed and the drying gas flow rate is increased, then one would expect the charge distribution to shift in the direction of more charges per ion.

The experiments confirm this prognosis. Figure 4 shows two mass spectra for cytochrome $c$ typical of those obtained in this study. Both were taken at the same flow rate for cooling gas, but the bath gas flow rate for the lower spectrum was higher than for the upper spectrum. There is clearly a substantial shift to lower mass-to-charge ratio values (more charges per ion) at the higher flow rate of drying gas. Figure 5 summarizes the effects due to variation in each of the two flow rates. The ordinate values show the number of charges on the most abundant ions-those in the highest peak. As the flow of concurrent cooling gas increases at a constant flow rate of drying gas, the number of charges on the highest peak decreases, indicating a shift in the distribution toward lower charge states. Conversely, increasing the flow of countercurrent drying gas at a constant flow of cooling gas increases the number of charges on the highest peak, indicating a shift in the distribution toward higher charge states. Some experiments were also carried out in which the temperature of the drying gas was varied. The results were not as unequivocally clear because the gas temperature could be varied only very slowly and not by very much. Nevertheless, it was clear that increasing its temperature did produce a bias toward the higher charge states, as should be the case if this temperature increase raised the evaporation rates as expected. These two sets of observations on the effect of changing droplet evaporation rates constitute strong evidence that the ions desorbing early in the droplet evaporation process do indeed have lower charge states, as the picture painted here would suggest.

\section{Conclusions}

The scenario described in this report does not pretend to comprise a complete or exact description of the process by which solute species in charged droplets become frce ions in the ambient gas. It does seem to provide at least a qualitative explanation for the general pattern of bell-shaped charge distributions that are characteristic of ES/MS experiments with large

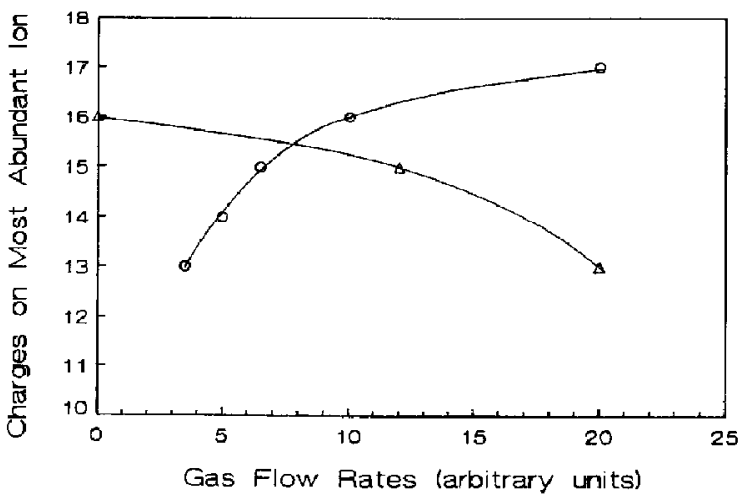

Figure 5. Summary of data taken at various combinations of flow rates for cooling and drying gases. The ordinate values of the circles represent the number of charges on the ions of the highest peaks in a series of spectra of which two are shown in Figure 3 (i.e., with increasing flow rate of bath gas). The triangles are from similar spectra obtained while the cooling gas flow rate was increasing at a constant flow rate of drying gas, thus decreasing the droplet evaporation rate and therefore the number of charges per ion. 
molecules. It also reveals for the first time the likelihood that in those distributions, the ions with few charges leave the droplet earlier in the evaporation sequence than those with many charges. Moreover, the scenario plausibly accounts for the observed differences in charge distribution that are found for molecules of the same species in different configurations. In a sense this account is a mirror image of the perception of previous investigators that such differences depend on whether in a folded molecule the charges have access to the sites. Here the question asked is whether the sites have access to the charges. It is the charges that are immobilized, so the mountain must go to Mohammed instead of vice versa.

Not to be forgotten, however, is that this scenario as well as others may be substantially distorted by a factor seldom mentioned-the response of detectors to multiply charged ions. Little information is available on the yield of secondary electrons when a large, multiply charged ion hits the surface of a dynode. Thus, an unanswered question is whether observed peak heights reflect the number of incident ions, the number of charges they carry, the energy of incidence, its velocity, or an unknown combination of these factors. Some very interesting results from the Sundquist group at Uppsala with regard to multiply charged protein ions at very high incident energies indicate that the secondary electron yield may even depend on the conformation of the incident ion [15]. Clearly, the usual tacit assumption, that peak heights in a mass spectrum are directly proportional to the number of ions incident on the detector, must remain suspect until the responses of these detectors have been appropriately characterized.

\section{Acknowledgments}

The author thanks Takashi Nohmi, J. Fred Banks, and Shida Shen for making some of their unpublished data available, as well as
Errol Gulcicek and Steve Fuerstenau for help in preparing the figures. In addition, he thanks these colleagues, along with Juan F. de la Mora, Craig Whitehouse, Alessandro Gomez, Joan Roselle-Llompart, and Ignacio Los Certales, for many helpful and provocative discussions. This work was supported in part by NIH grant R01 GM31660-07A1 and in part by NSF grant MCB9118224.

\section{References}

1. (a) Dole, M.; Mach, L. L.; Hines, R. L.; Mobley, R. C.; Ferguson, L. P.; Alice, M. B. J. Chem. Phys. 1968, 49, 2240; (b) Mach, L. L.; Kralik, P., Rheude, A., Dole, M. I. Chem. Phys. 1970, 52, 4977.

2. Zeleny, T. Phys. Rev. 1917, 10, 1.

3. Iribanne. J. V.; Thomson, B. A. J. Chern. Phys. 1976, 64, 2287.

4. (a) Thomson, B. A; Iribarne, J. V. I. Cham. Phys. 1979, 71, 4451; (b) Iribarne, J. V.; Dziedzic, P. J; Thomson, B. A. J. Mass Spectrom. Ion Phys. 1983, 50, 331.

5. (a) Blakley, C. R.; McAdams, M.; Vestal, M. L. J. Chronatogr. 1978, 158, 264; (b) Blakley, C. R.; Carmody, J. J; Vestal, M. L. Clin. Chem. 1980, 16, 1467; (c) J. Am. Chem. 5oc. 1980, 102, 5931.

6. Bruins, A. P.; Covey, T. R.; Henion, J. D. 1987, 59, 2642.

7. (a) Roellgen, F. W.; Bramer-Weger, E.; Buetfering, L. J. Phys. (Paris) 1987, 48, C6-253; (b) Schmelzeisen-Redeker, G.; Buetfering, L.; Roellgen, F. W. Int. I. Mass Spectrom. Ion Processes 1989, 90, 139.

8. Nohmi, T.; Fenn, J. B. J. Am. Chem. Soc. 1992, 114, 3241.

9. Taflin, D. C.; Ward, T. L.; Davis, E. J. Langmuir 1989, 5, 376.

10. Covey, T. R.; Bonner, R. F.; Shushan, B. I.; Henion, J. D. Rapid Commun. Mass Spectrom. 1988, 2, 249.

11. Wong, S. F.; Meng, C. K.; Fenn, J. B. J. Phys. Chem. 1988, 30, 368.

12. Loo, J. A.; Edmonds, C. D; Udseth, H. R; Smith, R. D. Anal. Chem. 1990, 62, 693.

13. Chowdhury, S. K.; Katta, V.; Chait, B. T. I. Am. Chem. Soc. 1990, 12, 9012

14. Guevremont, R; Siu, K. W. M.; Le Blanc, J. C. Y.; Berman, S. S. I. Am. Soc. Mass Spectrom. 1992, 3, 216.

15. Reimann, C. T. Uppsala University, private communication, 1992. 